\title{
Abstimmungskampf, Informationsvermittlung und Stimmentscheidung beim Volksentscheid über den Nicht- raucherschutz in Bayern
}

\author{
Harald Schoen/Alexander Glantz/Rebecca Teusch
}

\section{A. Einleitung}

Seit Beginn der Bundesrepublik drehen sich Diskussionen über direktdemokratische Verfahren nicht selten um die Rolle der Bürger darin. Ein geradezu klassischer Einwand gegen solche Verfahren lautet, etliche Bürger seien an politischen Fragen zu wenig interessiert und zu schlecht darüber informiert, als daß sie fundierte Entscheidungen über politische Sachfragen treffen könnten und sollten. Die resultierenden Entscheidungen würden daher der Komplexität vieler politischer Materien nicht gerecht. ${ }^{1}$ Dies könne so weit gehen, daß Bürger sich von Volksverführern zu fragwürdigen, ja gefährlichen Entscheidungen verleiten ließen, wie es Theodor Heuss in seinem vielzitierten Diktum von der „Prämie für jeden Demagogen“, die direktdemokratische Verfahren versprächen, formulierte. $^{2}$

Befürworter direktdemokratischer Verfahren suchen diesen Einwand mit verschiedenen Argumenten zu entkräften. Aus normativer Sicht wird die Frage aufgeworfen, ob hinreichende Informiertheit und Kompetenz als Voraussetzung für Entscheidungsrechte angesehen werden sollten. Damit verwandt ist das Argument, daß auch Parlamentarier nicht selten weitreichende Entscheidungen träfen, ohne hinreichend gut informiert zu sein. Ein direktdemokratisches Votum bringe daher zumindest keine Verschlechterung gegenüber Entscheidungen in repräsentativdemokratischen Gremien mit sich. Schließlich wird angeführt, direktdemokratische Verfahren milderten das (vermeintliche) Wissensdefizit der Bürger selbst ab. Denn diese Verfahren böten - anders als repräsentativdemokratische Verfahren - Bürgern Anreize, sich politisch sachkundig zu machen: Da sie selbst

1 Vgl. H. Oberreuter, Direkte Demokratie und die repräsentative Verfassung der Bundesrepublik Deutschland, in: ZfP 49 (2002), 290 (296 f.).

2 Siehe etwa F. Decker, Die Systemverträglichkeit der direkten Demokratie. Dargestellt an der Diskussion um die Einführung von plebiszitären Elementen in das Grundgesetz, in: ZPol 15 (2005), 1103 (1119 f.). 
entscheiden dürfen, würden sich die Bürger besser informieren. ${ }^{3}$ Darüber hinaus würden in den Kampagnen vor Volksabstimmungen Vertreter von Verbänden und Parteien sowie Einzelpersonen öffentlich die jeweilige Sachfrage aus verschiedenen Perspektiven beleuchten, Bürgern etliche Informationen bereitstellen und könnten sie sogar dazu anregen, „besser“, etwa im Sinne der eigenen sachbezogenen Präferenzen oder auf der Grundlage verallgemeinerbarer Argumente zu entscheiden. ${ }^{4}$

Empirische Analysen haben Anhaltspunkte dafür zusammengetragen, daß Kampagnen vor Volksabstimmungen tatsächlich derartige Wirkungen entfalten können. Es konnte gezeigt werden, daß im Laufe von Abstimmungskampagnen die Aufmerksamkeit und das Interesse von Bürgern an der Abstimmung und deren Gegenstand anwächst. ${ }^{5}$ Auch scheinen Bürger infolge der Kampagnen und der Medienberichterstattung hinzuzulernen, und zwar über die Inhalte der zur Abstimmung stehenden Vorlagen wie auch darüber, welche Positionen Parteien und Interessengruppen in der Auseinandersetzung einnehmen. ${ }^{6}$ Größere Aufmerksamkeit und zusätzliche Informationen können Bürger zur Teilnahme an der Abstimmung mobilisieren und zu Meinungsänderungen führen. ${ }^{7}$ Die in der Kampagnenphase zur Verfügung gestellten Informationen können Stimmbürgern zudem dabei behilflich sein, wohlbedachte Entscheidungen im Einklang mit ihren Interessen und sachbezogenen Grundorientierungen zu treffen. ${ }^{8}$ Zusammengenommen deutet die Evidenz somit darauf hin, daß Abstimmungskampagnen einen wichtigen Beitrag leisten können, die Zweifel an der Kompetenz von Bürgern zu zerstreuen.

3 Vgl. G. Kirchgässner/L.P. Feld/M.R. Savioz, Die direkte Demokratie. Modern, erfolgreich, entwicklungs- und exportfähig, 1999, S. 53 ff.

4 Vgl. etwa B.S. Frey/G. Kirchgässner, Diskursethik, Politische Ökonomie und Volksabstimmungen, in: Analyse und Kritik 15 (1993), $129 \mathrm{ff}$.

5 Vgl. etwa S. Bowler/T. Donovan, Information and opinion change on ballot propositions, in: Political Behavior 16 (1994), $411 \mathrm{ff}$.

6 Vgl. S. Bowler/T. Donovan, Do voters have a cue? Television advertisements as a source of information in citizen-initiated referendum campaigns, in: EuJPolR 41 (2002), 777 ff.;

M. Mendelsohn/F. Cutler, The effect of Referendums on Democratic Citizens: Information, Politicization, Efficacy and Tolerance, in: BJPS 30 (2000), 685 ff.

7 Vgl. Bowler/Donovan, Information (Fn. 5).

8 Vgl. etwa A. Lupia, Shortcuts versus encyclopedias: information and voting behavior in California insurance reform elections, in: APSR 88 (1994), 63 ff.; S. Bowler/T. Donovan, Demanding Choices. Opinion, Voting, and Direct Democracy, Ann Arbor 1998, Kap. 3, 7 , 8; H. Kriesi, Direct democratic choice: The Swiss experience, Lanham, 2005; S.B. Hobolt, Taking Cues on Europe? Voter competence and party endorsements in referendums on European integration, in: EuJPolR 46 (2007), 151 ff.; P. Selb/H. Kriesi/R. Hänggli/M. Marr, Partisan Choices in a Direct-Democratic Campaign, in: EuPolSR 1 (2009), 155 ff. 
Mit Blick auf direktdemokratische Verfahren in Deutschland haben diese Befunde den Schönheitsfehler, daß sie sich auf direktdemokratische Verfahren jenseits der deutschen Grenzen beziehen und wegen der Kontextabhängigkeit politischer Urteilsbildung und politischen Verhaltens nicht zuverlässig Aufschluß über die Verhältnisse in der Bundesrepublik erlauben. ${ }^{9}$ Wir versuchen daher mit dem vorliegenden Aufsatz dazu beizutragen, diese Lücke zu schließen. Wir untersuchen am Beispiel des Volksentscheids zum Nichtraucherschutz in Bayern, inwieweit die Kampagnen der Ja- und der Nein-Seite die Bürger erreichten, deren Interesse weckten oder verstärkten, gegenstands- und akteursbezogene Kenntnisse vermittelten und inwieweit Kenntnisse das Abstimmungsverhalten beeinflußten. Der Beitrag schließt mit einer kurzen Zusammenfassung und Diskussion zentraler Ergebnisse.

\section{B. Der Abstimmungskampf und seine Rezeption}

Der Abstimmungskampf vor dem Volksentscheid am 4. Juli 2010 war geprägt vom Konflikt zwischen zwei eigens zum Volksentscheid gegründeten Bündnissen. Auf der Seite der Befürworter eines strengeren Nichtraucherschutzes stand das „Aktionsbündnis Nichtraucherschutz Bayern“, das neben verschiedenen Verbänden ÖDP, SPD und Bündnis 90/Die Grünen vereinte und dessen öffentlich wohl sichtbarster Einzelakteur Sebastian Frankenberger war. Die Gegner des Volksentscheids hatten sich im „Aktionsbündnis für Freiheit und Toleranz“ (AFT) zusammengeschlossen. Beiden Seiten standen - verglichen mit Wahlkämpfen und, erst recht, mit den Budgets für kommerzielle Werbung - recht bescheidene finanzielle Ressourcen zur Verfügung. Mit ihren begrenzten finanziellen Mitteln führten beide Seiten eine engagierte Auseinandersetzung und warben um die Stimmen der Bürger. ${ }^{10}$ Mit wieviel Engagement Akteure auf beiden Seiten bei der Sache waren, läßt sich sehr gut auf den entsprechenden FacebookSeiten erkennen, die - vermutlich auch wegen finanzieller Restriktionen - in den Kampagnen als wichtige Kommunikations- und Koordinationsforen dienten.

9 Für die Bundesrepublik liegen bisher lediglich empirische Aggregatanalysen, etwa der Abstimmungskampfkommunikation, vor. Siehe hierzu V. Mittendorf, Die Qualität kollektiver Entscheidungen, 2009, S. $131 \mathrm{ff}$.

10 Siehe ausführlich zu Akteuren und Kampagnenführung B.M. Weixner, Nichtraucherschutz in Bayern - der Weg eines erfolgreichen Volksbegehrens und Volksentscheids im Freistaat, in diesem Band (oben S. 258 ff.). 
Eine engagiert geführte Kampagne garantiert allerdings keine große öffentliche Aufmerksamkeit und Resonanz. Um den öffentlichen Widerhall des Abstimmungskampfes zu untersuchen, greifen wir auf Daten aus einer Befragung zurück, die im Rahmen des DFG-Projekts „Politische Urteilsbildung in direktdemokratischen Verfahren. Eine Analyse zum Volksentscheid über den Nichtraucherschutz in Bayern 2010“ durchgeführt wurde. Zwischen dem 25. Mai und dem 3. Juli 2010 wurden insgesamt 4000 zufällig ausgewählte stimmberechtigte Bayern zum Volksentscheid telefonisch (CATI) befragt. Es wurde das sogenannte Rolling-Cross-Section-Design ${ }^{11}$ gewählt, d.h. die 4000 Befragten wurden (annähernd) zufällig so auf die vierzig Tage vor dem Volksentscheid verteilt, daß täglich hundert von ihnen befragt wurden. Im Ergebnis erlauben es unsere Daten, die öffentliche Resonanz der Kampagnen und die Meinungsbildung der Bürger in den letzten vierzig Tagen vor dem Volksentscheid zu untersuchen.

Auf der Grundlage dieser Daten haben wir den Anteil der Befragten ermittelt, die im Untersuchungszeitraum angaben, mit den Kampagnen der Ja- oder der Nein-Seite direkt in Berührung gekommen zu sein (Abbildung 1a-f). Demnach stieg der Anteil der Personen, die von Plakaten berichteten, deutlich von anfangs etwa zwanzig auf über fünfzig Prozent an. ${ }^{12}$ Auch bei der Werbung für oder gegen den Gesetzentwurf „Für echten Nichtraucherschutz!“ in Presse, Fernsehen oder Radio läßt sich ein erheblicher Anstieg auf rund vierzig Prozent erkennen. Von Flugblättern und ähnlichen Werbematerialien berichteten anfangs praktisch keine Befragten, unmittelbar vor dem Volksentscheid jedoch rund ein Fünftel von ihnen. Persönliche Kontakte zu Befürwortern oder Gegnern des Gesetzentwurfs „Für echten Nichtraucherschutz!“, etwa im Straßenabstimmungskampf, scheinen nur sehr selten vorgekommen zu sein, und daran änderte sich im Kampagnenverlauf praktisch nichts. Ein ähnliches Muster zeichnet sich für die Nutzung des Internets sowie die Rezeption einschlägiger E-Mails und SMS ab. Die engagiert geführten Kampagnen auf den Facebook-Seiten scheinen somit nur einen sehr kleinen Teil der Bevölkerung direkt erreicht zu haben.

11 Siehe dazu etwa R. Johnston/H. Brady, The Rolling Cross-Section Design, in: Electoral Studies 21 (2002), 283 ff.; R. Schmitt-Beck/T. Faas/C. Holst, Der Rolling Cross-Section Survey - ein Instrument zur Analyse dynamischer Prozesse der Einstellungsentwicklung, in: ZUMA-Nachrichten 58 (2006), $13 \mathrm{ff}$.

12 Um die Darstellung übersichtlich zu gestalten, verzichten wir darauf, die Ergebnisse dem komplexen Erhebungsdesign methodisch angemessener Regressionsanalysen eigens zu berichten. Sie fließen jedoch insofern in die Darstellung mit ein, als wir nur solche Veränderungen herausstellen, die sich in diesen Analysen als statistisch signifikant und substantiell relevant erwiesen. 
Abbildung 1: Häufigkeit verschiedener Kampagnenkontakte in den letzten 40 Tagen vor dem Volksentscheid
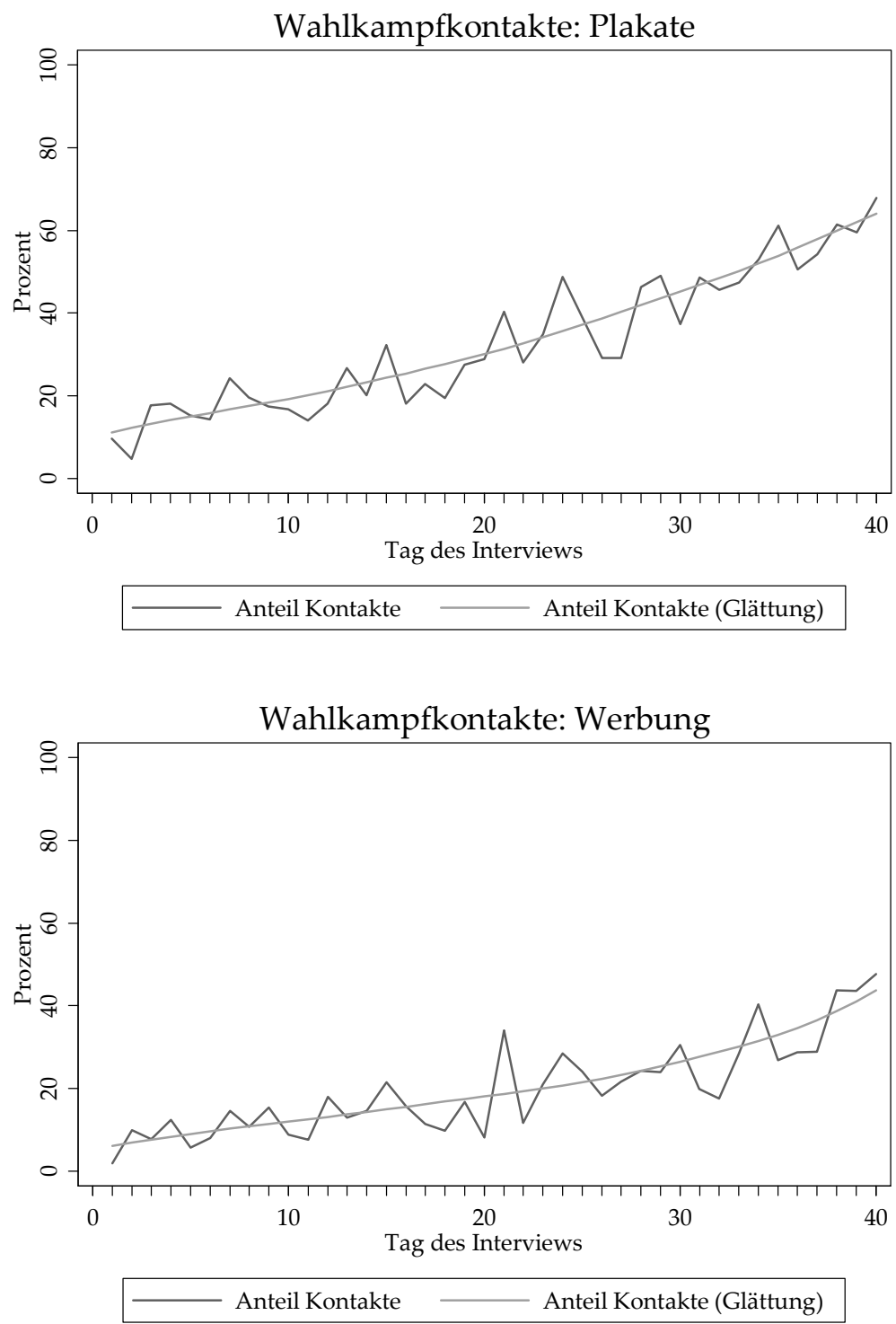


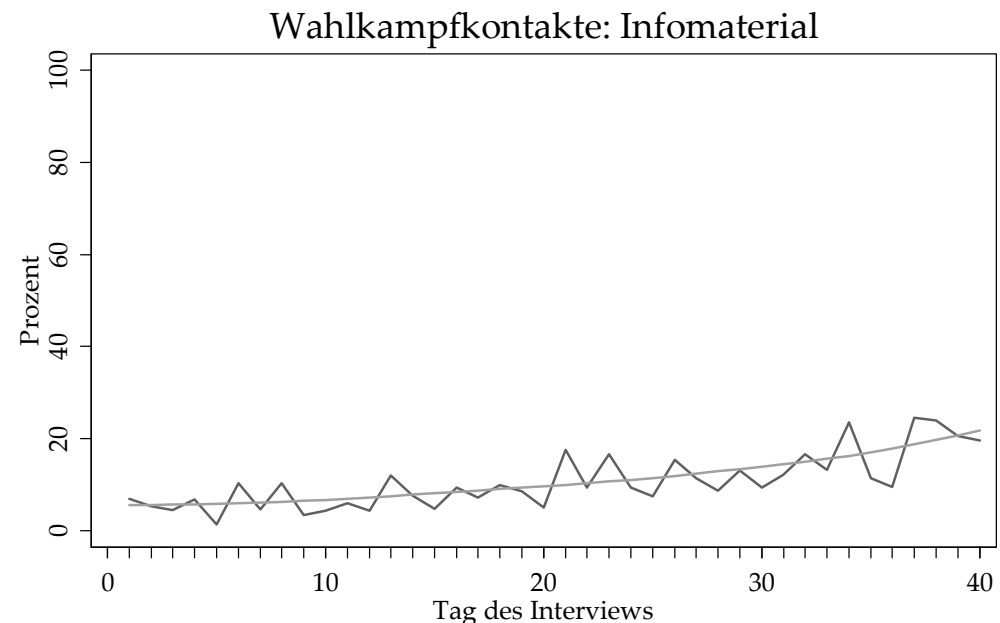

Anteil Kontakte

Anteil Kontakte (Glättung)

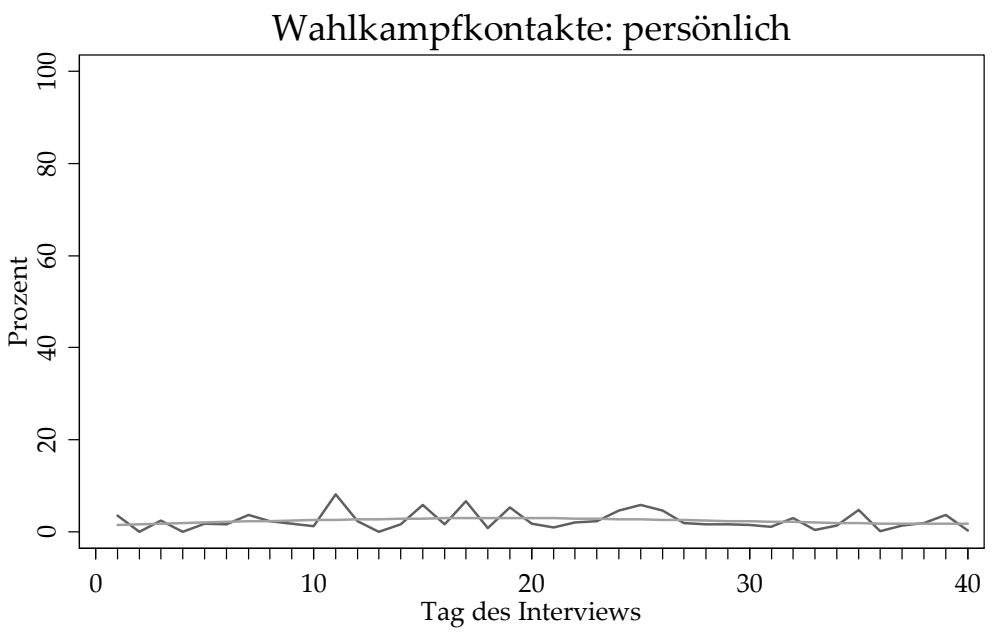

Anteil Kontakte

Anteil Kontakte (Glättung) 


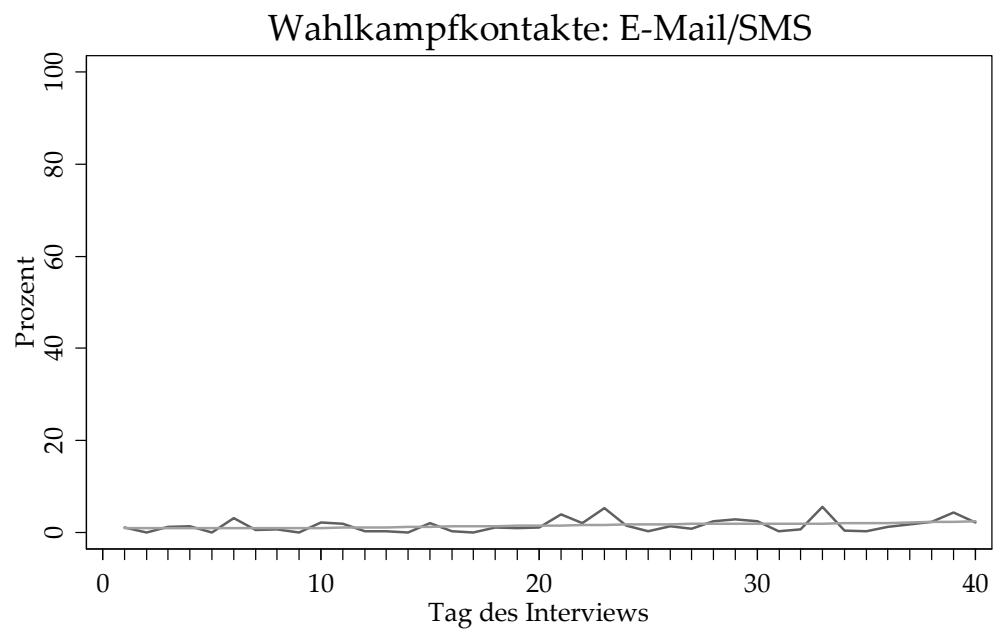

Anteil Kontakte

Anteil Kontakte (Glättung)

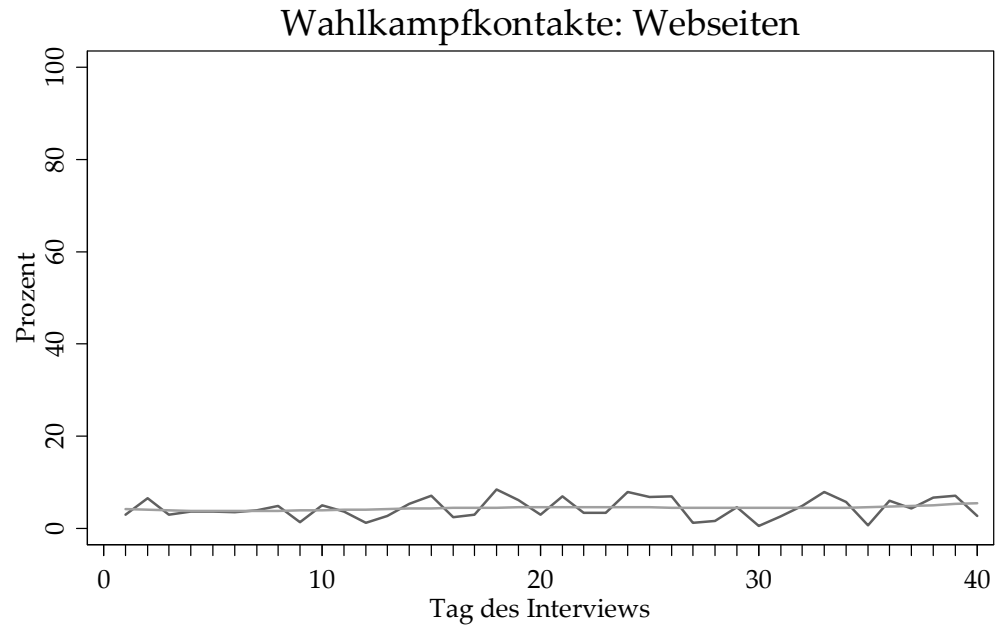

Anteil Kontakte

Anteil Kontakte (Glättung)

Anmerkung: Der erste Befragungstag ist der 25. Mai 2010, der 40. Befragungstag der 3. Juli 2010. 
Diese Befunde deuten auf eine gewisse Reichweite des Abstimmungskampfes hin. Sie wurde dadurch unterstützt, daß 20 Prozent der Befragten - wie weitere Analysen zeigen - auf den Volksentscheid bezogene Inhalte in den Nachrichtensendungen des Bayerischen Rundfunks entdeckten, 60 Prozent in den Tageszeitungen. Zugleich darf die Reichweite keinesfalls überschätzt werden. So belegen detaillierte Analysen, daß nur wenige Befragte Kampagnenkontakte zu verschiedenen Organisationen, also etwa die Rezeption von Werbung der Ja- und der Nein-Seite, angaben (nicht graphisch ausgewiesen). Aus der Sicht vieler Stimmberechtigter erreichte der Abstimmungskampf somit eine allenfalls mäßige Intensität. Angesichts der vergleichsweise bescheidenen Mittel beider Seiten und des gesellschaftlichen Umfeldes - der Volksentscheid konkurrierte mit der Bundespräsidentenwahl und der Fußballweltmeisterschaft um öffentliche Aufmerksamkeit - scheint dieses Ergebnis folgerichtig. Zugleich deutet es darauf hin, daß die Kampagnen nicht viel dazu beitragen konnten, das Interesse vieler Stimmberechtigter am Volksentscheid zu wecken oder zu verstärken und ihnen darauf bezogene Informationen zu vermitteln. Diesen Fragen wenden wir uns nun zu.

\section{Interesse, Kenntnisse und Stimmverhalten der Bürger}

Der Volksentscheid am 4. Juli 2010 stieß bei den Stimmberechtigten im Durchschnitt auf ein mittleres Interesse (Abbildung 2). Während der heißen Kampagnenphase stieg der Anteil der ziemlich oder sehr stark interessierten Bürger merklich an. Allerdings fiel der Anstieg mit rund zehn Prozentpunkten nicht übermäßig stark aus. Ebensowenig sorgte er dafür, daß unmittelbar vor der Abstimmung alle Stimmberechtigten hochgradig interessiert gewesen wären. ${ }^{13}$

13 Das allgemeine politische Interesse blieb während des Untersuchungszeitraums praktisch konstant und war anfangs deutlich größer als das Interesse am Volksentscheid, jedoch nicht mehr am Ende der Kampagne. 


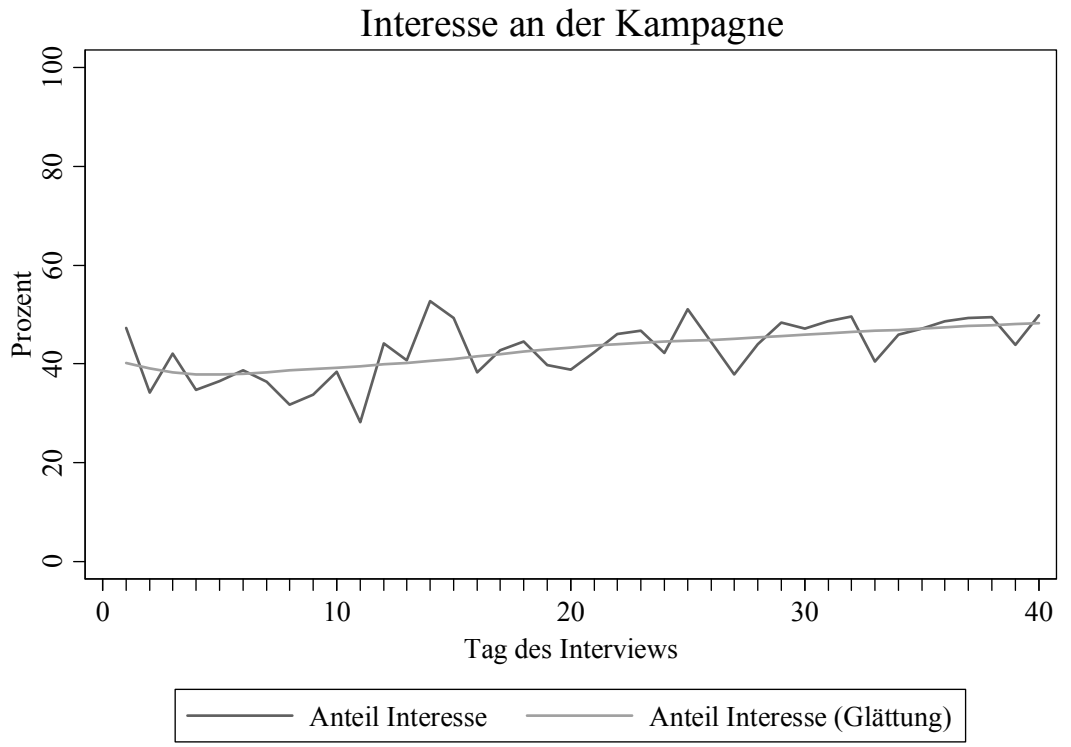

Anmerkung: Der erste Befragungstag ist der 25. Mai 2010, der 40. Befragungstag der 3. Juli 2010.

Gleichwohl könnte das gewachsene Interesse an der Volksabstimmung Stimmberechtigte dazu motiviert haben, sich über den zur Abstimmung stehenden Gesetzentwurf sowie die Kampagnenakteure auf beiden Seiten zu informieren. Folglich wäre damit zu rechnen, daß die Stimmberechtigten im Laufe der Kampagne zunehmend besser Bescheid wußten. Diese Erwartung erscheint noch plausibler, wenn man die weiter oben dargestellte selektiv gewachsene Reichweite der Kampagnen und die Medienberichterstattung bedenkt, die auch Informationen verbreiteten.

Unsere Daten erlauben es, diese Vermutung für gegenstands- und akteursbezogenes Wissen getrennt $\mathrm{zu}$ untersuchen. Um gegenstandsbezogenes Wissen zu messen, wurden die Respondenten zum einen nach dem Termin des Volksentscheids gefragt. Zum anderen wurden sie gebeten, fünf Aussagen zum 
Gesetzentwurf als richtig oder falsch einzuordnen. ${ }^{14}$ Wie Abbildung 3a-f zeigt, war es für die Respondenten anfangs offenbar sehr schwierig, den Termin des Volksentscheids zu nennen. Binnen vierzig Tagen stieg der Anteil richtiger Antworten allerdings rapide von anfangs weniger als zehn Prozent auf etwa 80 Prozent an. Dieser Anstieg zeigt, wie stark die Verbreitung politischer Kenntnisse während der Kampagne unter den gegebenen gesellschaftlichen und politischen Bedingungen zunehmen konnte. Es handelt sich um eine sehr einfache und daher leicht vermittelbare Information, die von Kampagnenakteuren, Massenmedien und staatlichen Stellen - in Form von Wahlbenachrichtigungen - an die Stimmberechtigten weitergegeben wurde. Angesichts dieser günstigen Bedingungen dürfte der Anstieg den empirisch maximal möglichen Effekt auf die politischen Kenntnisse markieren.

Bei den anderen Fragen, die sich auf kompliziertere oder weniger prominent vermittelte Sachverhalte beziehen, sind erwartungsgemäß deutlich weniger Veränderungen im Kenntnisstand erkennbar. Die Ergebnisse zu den Raucherräumen in Krankenhäusern und zur Haftung von Gaststättenbetreibern blieben - auf deutlich unterschiedlichen Niveaus - im Untersuchungszeitraum praktisch vollkommen unverändert. In bezug auf die im Gesetzentwurf angeblich vorgesehenen Haftstrafen nimmt der Anteil richtiger Antworten sogar leicht ab. Bei den beiden verbleibenden Fragen können wir hingegen einen deutlichen, wenn auch nicht übermäßig starken Anstieg beobachten. Beantwortete am Anfang des Beobachtungszeitraums rund die Hälfte der Respondenten die Frage nach dem Rauchen in Bierzelten richtig, so lag dieser Anteil unmittelbar vor der Abstimmung bei knapp 70 Prozent. Der Anteil derjenigen Befragten, die die Frage nach den Abstimmungsregeln richtig beantworteten, stieg im Beobachtungszeitraum von rund einem Viertel auf etwa ein Drittel an.

14 Richtige Antworten auf diese Fragen können Wissen oder aber auch erfolgreiches Raten indizieren. Soweit letzteres zutrifft, überschätzen die ausgewiesenen Werte den tatsächlichen Wissensstand. 
Abbildung 3: Anteil der Befragten mit richtigen Angaben zu sechs volksentscheidsbezogenen Fragen in letzten 40 Tagen vor dem Volksentscheid
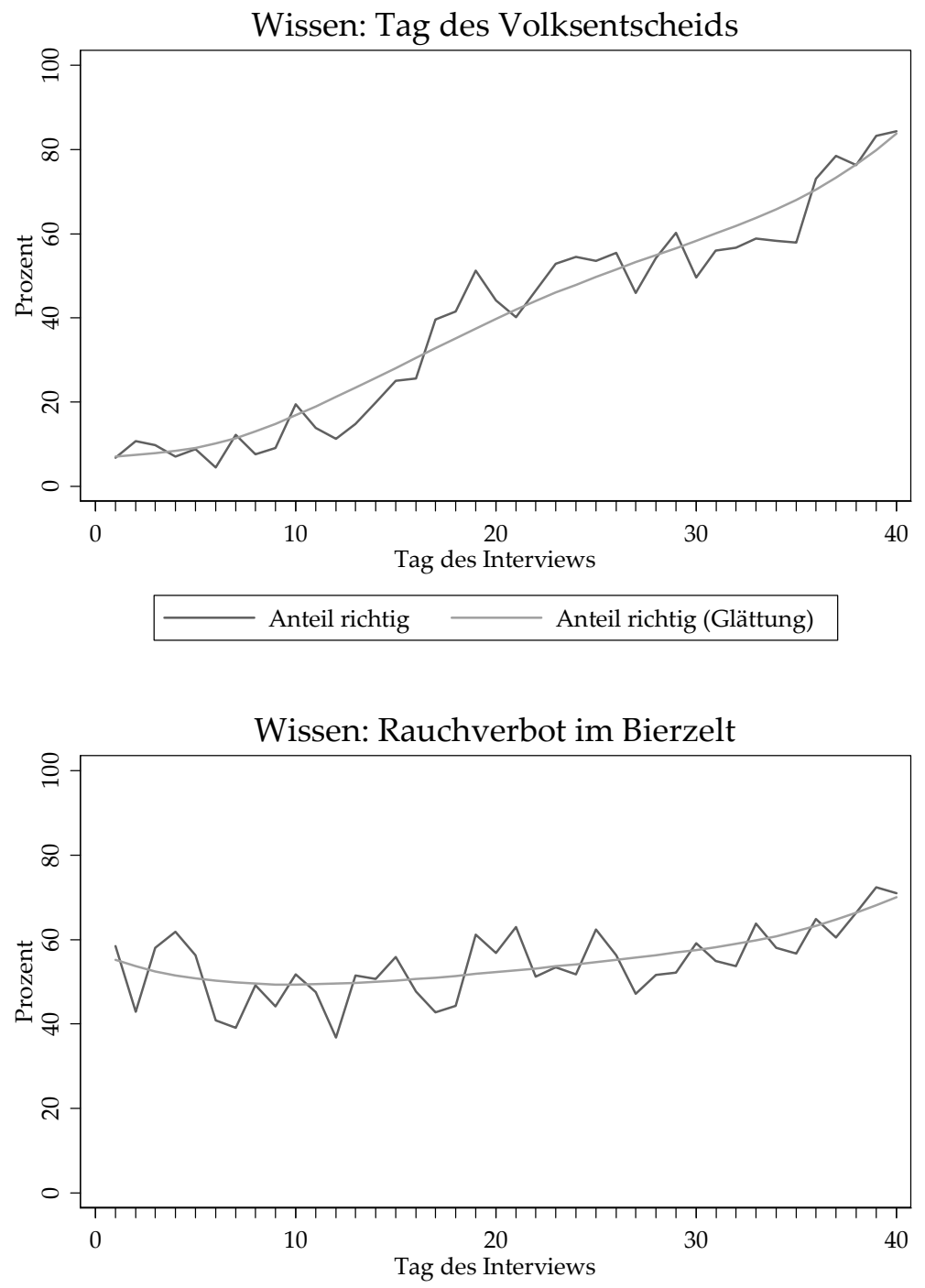

Anteil richtig Anteil richtig (Glättung) 


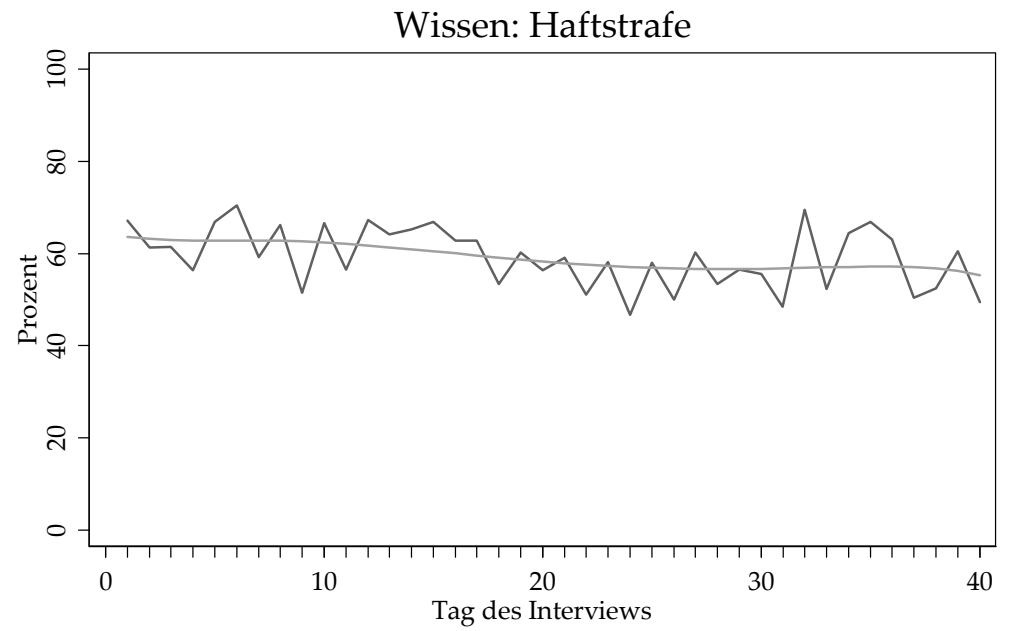

Anteil richtig

Anteil richtig (Glättung)

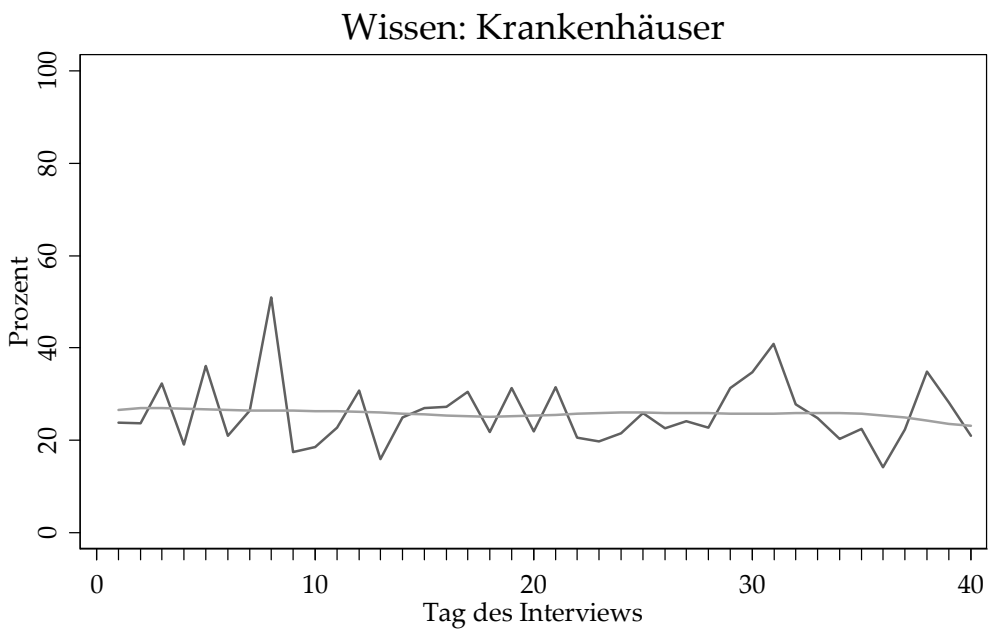

Anteil richtig

Anteil richtig (Glättung) 
Wissen: Gaststättenbetreiber

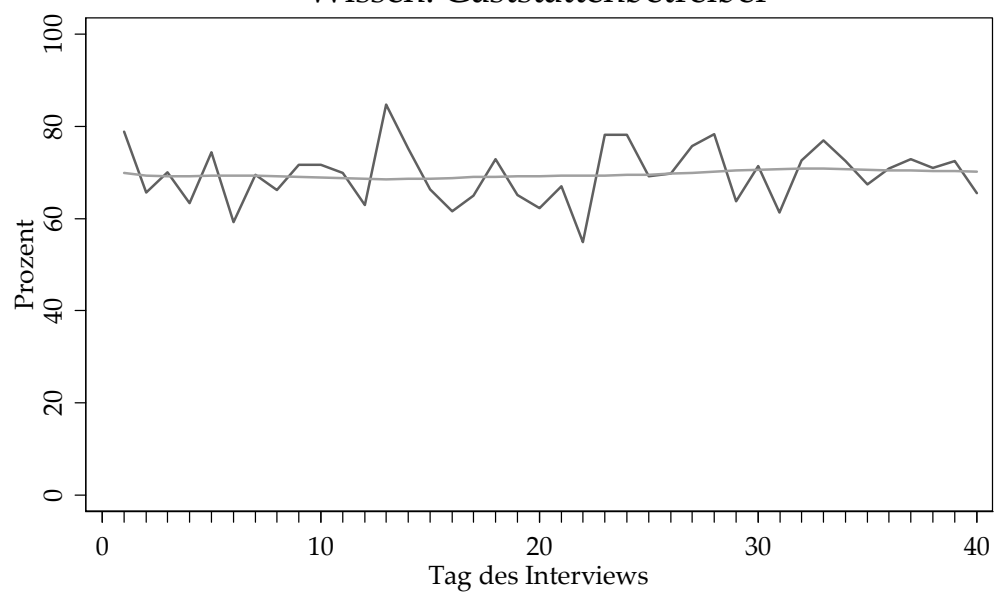

Anteil richtig

Anteil richtig (Glättung)

Wissen: Abstimmungsmodalität

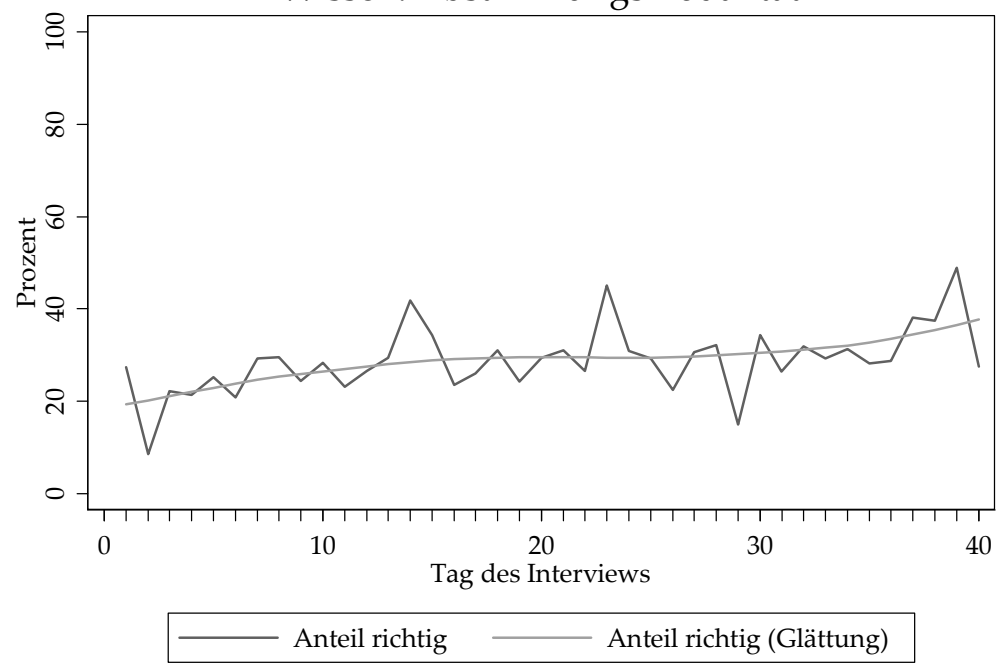

Anmerkung: Der erste Befragungstag ist der 25. Mai 2010, der 40. Befragungstag der 3. Juli 2010. 
Zusammengenommen scheinen die sachfragen- und verfahrensbezogenen Kenntnisse der Stimmbürger in der heißen Kampagnenphase eher selektiv denn umfassend zugenommen zu haben. Im Ergebnis wußte selbst am Tag vor der Abstimmung offenbar jeder vierte Stimmberechtigte nicht, daß ein Rauchverbot in Bierzelten zur Abstimmung stand. Ebenso war weniger als vierzig Prozent der Stimmberechtigten bewußt, daß die Nichtteilnahme am Volksentscheid nicht als Nein-Stimme wirken konnte. Somit konnten die Stimmberechtigten am 4. Juli in einigen Fragen als besser informiert gelten als noch ein paar Wochen vorher, von einem sachlich vollständig informierten Elektorat kann man aber schwerlich sprechen.

Neben sachfragen- und verfahrensbezogenem Wissen können Bürger bei direktdemokratischen Entscheidungen von akteursbezogenem Wissen profitieren. Wenn sie beispielsweise einschätzen können, welche gesellschaftlichen Gruppen oder Parteien auf welcher Seite der Auseinandersetzung stehen, können sie daraus unter Umständen zutreffende Schlußfolgerungen darüber ableiten, welches Votum am besten ihren Interessen diente. ${ }^{15}$ Die öffentliche Auseinandersetzung vor dem Volksentscheid am 4. Juli hätte daher den Stimmbürgern entsprechende Informationen bereitstellen und dadurch bei der Entscheidung helfen können.

Die Voraussetzungen dafür, daß sich Stimmberechtigte an den Standpunkten orientieren konnten, um ein ,richtiges“ Votum abzugeben, waren nicht ideal. Die CSU und die Freien Wähler (FW) verzichteten darauf, sich in der Auseinandersetzung um den Nichtraucherschutz eindeutig auf die eine oder andere Seite zu schlagen. Diese Entscheidungen lassen sich politisch gut begründen, doch führten sie dazu, daß den Stimmberechtigten zwei mögliche Orientierungspunkte fehlten. Es kommt hinzu, daß die Nicht-Positionierung von CSU und FW vielen Bürgern nicht klar war. Immerhin ein Viertel der Befragten glaubte, die Freien Wähler plädierten für einen strikteren Nichtraucherschutz, während etwa jeder zehnte Respondent den Freien Wählern die gegenteilige Position zuschrieb. Über die Haltung der CSU zum Volksentscheid herrschte unter den Stimmbürgern offenbar noch weniger Klarheit. So meinte immerhin knapp ein Drittel der Befragten, die CSU trete für eine Verschärfung des Nichtraucherschutzes ein, während gut ein Viertel der Respondenten die größere Regierungspartei auf der NeinSeite wähnte (nicht graphisch ausgewiesen). Diese wahrgenommenen Standpunkte konnten zwar subjektiv bei der Stimmentscheidung helfen, aber gewiß nicht zu einer ,richtigen“ Entscheidung führen, da sie den tatsächlichen Positionen von CSU und FW nicht entsprachen. 
Die anderen im Landtag vertretenen Parteien legten sich in der Auseinandersetzung zwischen Befürwortern und Gegnern eines strikteren Nichtraucherschutzes eindeutig fest. Sie hätten daher den Stimmberechtigten bei der Orientierung und Entscheidung behilflich sein können - vorausgesetzt, die Bürger nahmen die Standpunkte der Parteien zutreffend wahr. Wie Abbildung 4 a-f zu entnehmen ist, war diese Bedingung jedoch nur in gewissem Maße erfüllt. Rund sechzig Prozent der Befragten wußten, daß die bayerischen Grünen für ein strikteres Rauchverbot eintraten, etwa vierzig Prozent der Respondenten schrieb der SPD diese Position zu. Obwohl die SPD und die Grünen die Kampagne der Ja-Seite organisatorisch mittrugen, waren ihre Positionen nur etwas weniger bzw. etwas mehr als der Hälfte der Stimmberechtigten geläufig. Die FDP stand als einzige im Landtag vertretene Partei eindeutig auf der Seite der Gegner eines verschärften Nichtraucherschutzes, was jedoch weniger als einem Drittel der Befragten bekannt war. Diese Befunde dürften mit dem eher begrenzten Engagement der Landtagsparteien im Abstimmungskampf zusammenhängen.

Neben den im Landtag vertretenen Parteien hätten außerparlamentarische Kräfte den Bürgern bei der Entscheidung helfen können, die für oder gegen die geplante Verschärfung des Nichtraucherschutzes eintraten. Auf der Seite der Befürworter ist an erster Stelle an die ÖDP zu denken, die das Volksbegehren zum Nichtraucherschutz initiiert hatte. Wie die Ergebnisse zeigen, wußten allerdings nur knapp vierzig Prozent der Stimmberechtigten, daß die ÖDP für ein strikteres Rauchverbot eintrat. Noch schlechter informiert zeigten sich die Befragten über Sebastian Frankenberger, der gleichsam zum Gesicht der Ja-Seite avancierte. Denn während der letzten Wochen vor dem Volksentscheid wußten weniger als zehn Prozent der Befragten, daß Frankenberger sich für ,echten“ Nichtraucherschutz aussprach. Kaum anders sieht die Evidenz zum eigens mit Blick auf den Volksentscheid gegründeten „Aktionsbündnis für Freiheit und Toleranz“ aus: Weniger als zwanzig Prozent der befragten Bayern wußten, daß die zentrale Organisation der Nein-Seite sich gegen eine Gesetzesverschärfung einsetzte. Folglich konnten sich nur wenige Stimmberechtigte sinnvoll an diesen Organisationen und Personen bei der Stimmabgabe orientieren. 
Abbildung 4: Anteil der Befragten mit richtigen Angaben zu den Standpunkten von Parteien, Personen und Verbänden in den letzten 40 Tagen vor dem Volksentscheid
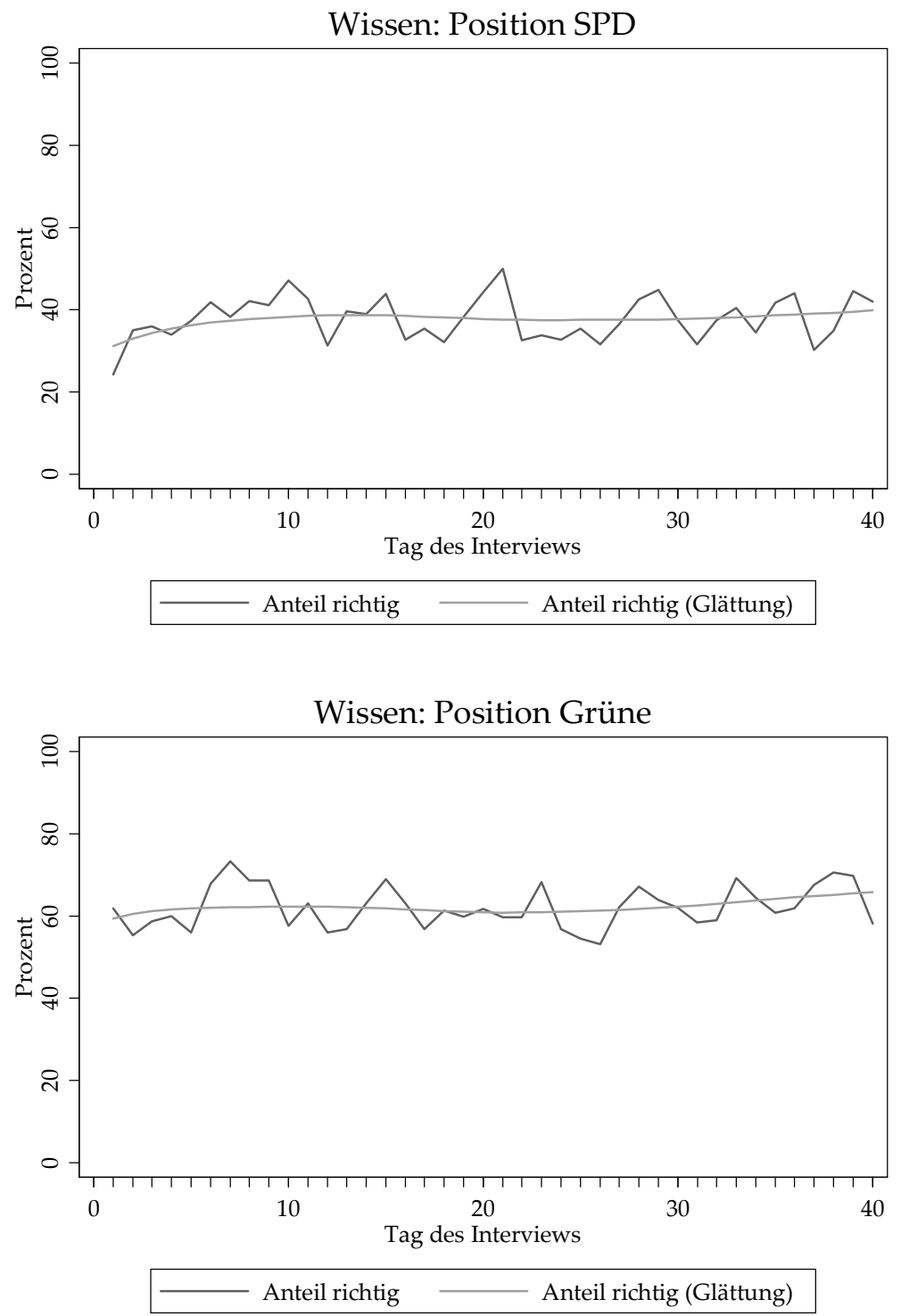

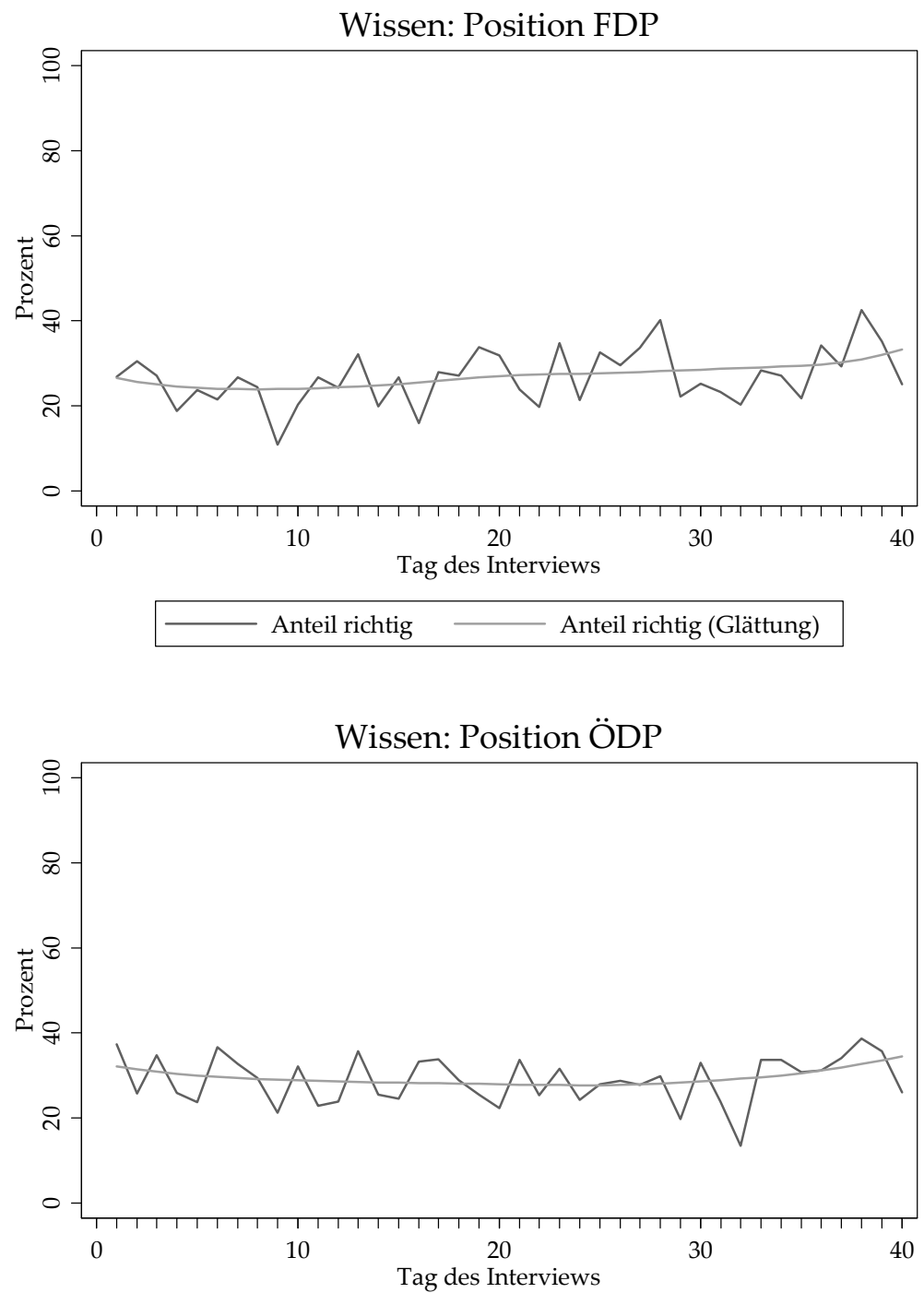

Anteil richtig

Anteil richtig (Glättung) 
Wissen: Position AFT

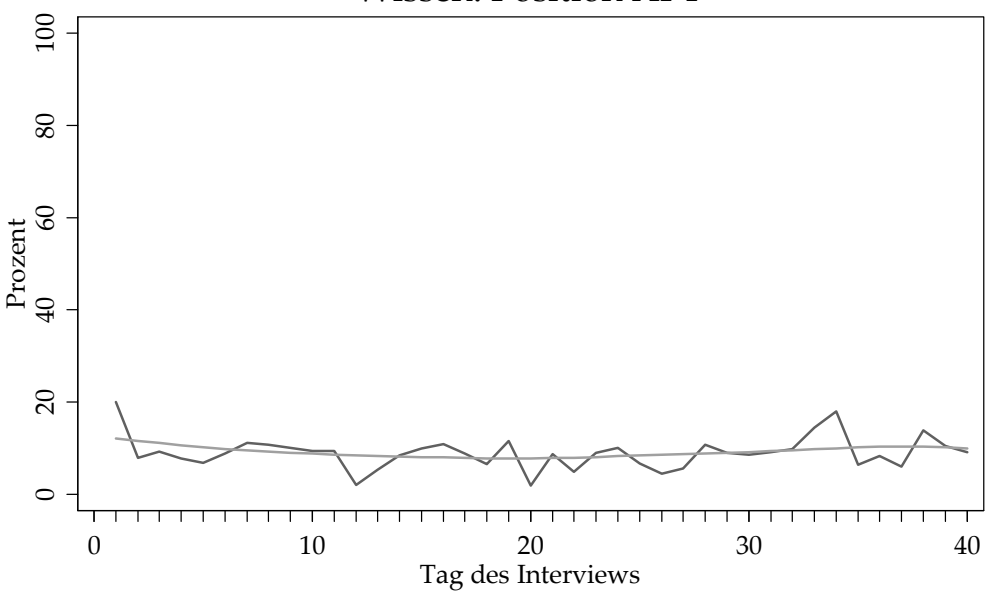

Anteil richtig

Anteil richtig (Glättung)

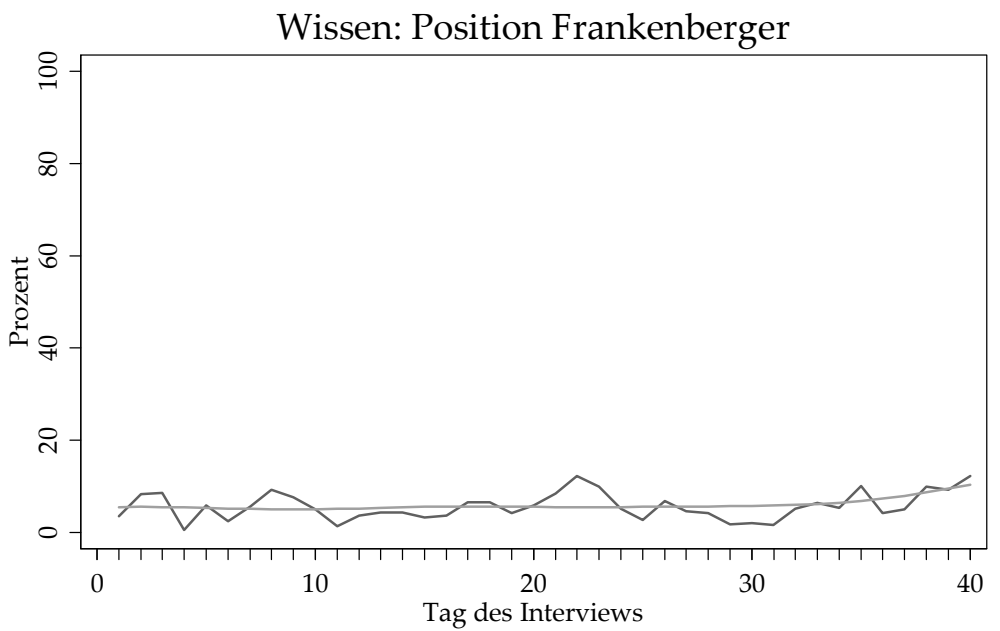

Anteil richtig

Anteil richtig (Glättung)

Anmerkung: Der erste Befragungstag ist der 25. Mai 2010, der 40. Befragungstag der 3. Juli 2010. 
Die in Abbildung 4a-f zusammengestellten Befunde zeigen darüber hinaus sehr deutlich, daß der Wissensstand der Bürger während der heißen Kampagnenphase praktisch vollkommen unverändert blieb. Folglich gelang es offenbar nicht, mittels Kampagnenkommunikation Informationen über die Standpunkte der verschiedenen individuellen und kollektiven Akteure weiterzuverbreiten. Im Lichte der oben beschriebenen geringen Kampagnenintensität betrachtet, kann dieses Ergebnis kaum überraschen. Zugleich bedeutet es jedoch, daß der Abstimmungskampf der Ja- und der Nein-Seite wenig dazu beitrug, den Bürgern sachfragen- oder akteursbezogene Informationen nahezubringen und dabei zu helfen, „richtig“" zu entscheiden. ${ }^{16}$

Inwiefern politische Kenntnisse das Stimmverhalten beeinflußten und daher ein besserer Kenntnisstand den Ausgang des Volksentscheids verändert hätte, ist empirisch zu klären. Dazu haben wir mit Hilfe einer logistischen Regressionsanalyse Determinanten der Stimmentscheidung untersucht. Neben einer Reihe sozialstruktureller Merkmale, dem Raucherstatus, dem Ausgehverhalten und der Parteibindung betrachten wir die (wahrgenommene) Sachfragenposition der Partei, mit der sich ein Respondent identifiziert, die Einstellung zum Nichtraucherschutz und zu staatlicher Intervention sowie schließlich die Kenntnis der Positionen von sechs Parteien zum Gesetzentwurf, der am 4. Juli 2010 zur Abstimmung stand, als potentielle Einflußgrößen. Die in Tabelle 1 zusammengestellten Ergebnisse zeigen, daß eine positive Haltung zum Nichtraucherschutz und zu staatlicher Intervention Ja-Voten begünstigte. Es fällt zudem auf, daß sich Männer und Raucher selbst unter Kontrolle dieser Einstellungen als überdurchschnittlich kritisch gegenüber dem Gesetzentwurf erwiesen.

Tabelle 1: Wirkung von politischen Orientierungen, politischen Kenntnissen und Kontrollvariablen auf die Stimmentscheidung (logistische Regression)

\begin{tabular}{l|cc}
\hline & \multicolumn{2}{|c}{ Präferenz } \\
\hline Unabhängige Variablen & B & Standardfehler \\
Einstellung: Nichtraucherschutz & $7.09^{* * *}$ & 0.62 \\
Einstellung: Rolle des Staates & $2.24 * * *$ & 0.44 \\
\hline
\end{tabular}

16 Weiterführende Analysen zeigen, daß die vorgestellten Befunde auf Personen, die an der Abstimmung teilnahmen, und solche, die keine Stimme abgaben, in sehr ähnlichem Maße zutreffen, auch wenn erstere Gruppe in einzelnen Hinsichten geringfügig besser informiert gewesen zu sein scheint als letztere. 


\begin{tabular}{|c|c|c|}
\hline Elitensignal: Identifikationspartei & 0.20 & 0.12 \\
\hline Positionswissen: Parteien & $2.02 * * *$ & 0.51 \\
\hline $\begin{array}{l}\text { Nichtraucherschutz x } \\
\text { Positionswissen }\end{array}$ & $13.30 * * *$ & 2.93 \\
\hline Staatsrolle $\mathrm{x}$ Positionswissen & 1.63 & 2.04 \\
\hline Elitensignal x Positionswissen & -0.24 & 0.52 \\
\hline Raucher & $-1.51 * * *$ & 0.22 \\
\hline Gaststättenbesucher & -0.28 & 0.20 \\
\hline Parteibindung: CSU/FDP & 0.16 & 0.21 \\
\hline Alter & -0.01 & 0.01 \\
\hline Geschlecht (Männer) & $-0.82 * * *$ & 0.19 \\
\hline Bildung & -0.09 & 0.13 \\
\hline Konstante & $1.98 * * *$ & 0.25 \\
\hline$\%$ korrekt klassifiziert & 85 & \\
\hline Pseudo- $R^{2}$ (McFadden) & .43 & \\
\hline $\mathbf{N}$ & 1706 & \\
\hline
\end{tabular}

Ausgewiesen sind die unstandardisierten Koeffizienten und die Standardfehler. Alle kontinuierlichen Variablen wurden auf den Bereich 0-1 normiert und zentriert. Signifikanzniveaus: $* * * p<.001$.

Daneben spielen politische Kenntnisse eine zweifache Rolle. Zum einen stimmten besser informierte Personen - ceteris paribus - überproportional häufig mit „Ja“. Zum anderen beeinflussen die parteienbezogenen Kenntnisse die Stärke der von den Einstellungsgrößen ausgehenden Wirkungen auf die Stimmabsicht. Bei gut informierten Personen schlug die Haltung zum Nichtraucherschutz wesentlich stärker auf die Stimmabsicht durch als bei weniger gut informierten Bürgern. Um diesen Befund anschaulicher darzustellen, als es logistische Regressionskoeffizienten gestatten, haben wir aus den Regressionsergebnissen errechnet, wie stark die Einstellung zum Nichtraucherschutz die Wahrscheinlichkeit einer Ja-Stimme beeinflußte. Abbildung 5 zeigt, daß selbst bei geringen Kenntnissen 
ein Votum für den Gesetzentwurf bei einer zunehmend positiven Haltung zum Nichtraucherschutz wahrscheinlicher wurde. Allerdings verstärkten parteibezogene Kenntnisse diese Wirkung deutlich. Im Ergebnis orientierten sich sehr gut informierte Bürger bei der Entscheidung noch deutlich stärker als andere an ihrer Haltung zum Nichtraucherschutz.

Abbildung 5: Wirkung der Einstellung zum Nichtraucherschutz auf die Wahrscheinlichkeit einer Ja-Stimme in Abhängigkeit von politischen Kenntnissen

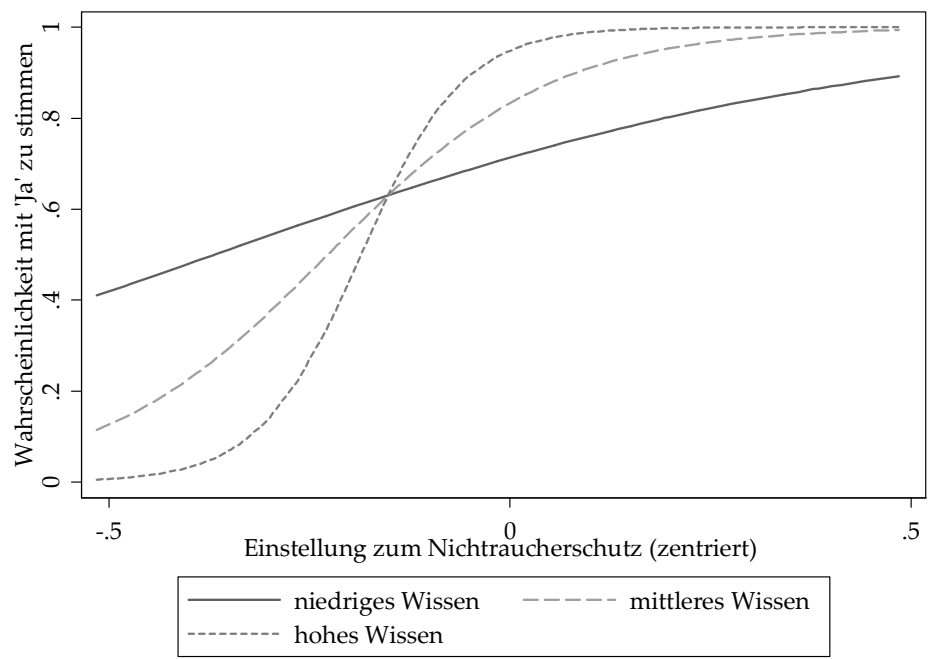

Anmerkung: Die Wahrscheinlichkeiten wurden aus den in Tabelle 1 dargestellten Ergebnissen berechnet. Die anderen im Modell enthaltenen Prädiktoren wurden auf den angemessenen Mittelwert gesetzt.

Parteienbezogene Kenntnisse hatten einen Einfluß darauf, wie sich die einzelnen Bürger entschieden, da sie die Entscheidung offenbar stärker als ein Votum für oder gegen Nichtraucherschutz erscheinen ließen. Daher hätten bessere Kenntnisse das individuelle Kalkül und die resultierende Stimmentscheidung verändern können. Allerdings hätten sie - unter sonst gleichen Bedingungen - das politische Ergebnis des Volksentscheids nicht wesentlich verändert. Denn weiterführende Simulationsrechnungen zeigen, daß ein besser informiertes Elektorat ceteris paribus - mit noch größerer Mehrheit für den Gesetzentwurf gestimmt hätte. 


\section{Schlußfolgerungen}

Dieser Beitrag untersuchte die Kampagnenrezeption, gegenstandsspezifisches Interesse und Wissen sowie das Entscheidungsverhalten beim Volksentscheid zum Nichtraucherschutz in Bayern. Die Analyse hat gezeigt, daß der Abstimmungskampf eine mäßige Reichweite erzielte und ein moderates Echo in den Medien und bei den Bürgern fand. In den letzten Wochen vor der Abstimmung stieg das Interesse der Bürger am Volksentscheid mäßig an, wie auch die politischen Kenntnisse der Bürger nur selektiv zunahmen. Letzteres ist umso bedeutsamer, als einschlägige Kenntnisse bei einigen Bürgern zu verändertem Stimmverhalten hätten führen können: Sie hätten stärker in Einklang mit ihren sachfragenbezogenen Grundorientierungen votiert und damit - in gewissem Sinne stärker interessenkonform gestimmt. Am Sieg der Ja-Seite hätten bessere Kenntnisse nichts geändert, im Gegenteil, sie hätten ihn - soweit die vorliegenden Daten darüber Aufschluß zu geben vermögen - noch deutlicher ausfallen lassen.

Betrachtet man den Volksentscheid über den Nichtraucherschutz in Bayern als ein Beispiel für direktdemokratische Verfahren in Deutschland, legt die Evidenz den Schluß nahe, daß direktdemokratische Kampagnen in Deutschland nicht zwangsläufig große öffentliche Aufmerksamkeit auf sich zu ziehen und allen Stimmberechtigten (oder auch nur allen Abstimmenden) relevante Informationen $\mathrm{zu}$ vermitteln vermögen. Abstimmungskampagnen sollten daher besser nicht als Allheilmittel angepriesen werden, die unter allen Umständen höchste Erwartungen an ihre Aufklärungsfunktion vor Volksabstimmungen erfüllen.

Dieser Befund ist per se kein Argument gegen Volksentscheide. Vielmehr legt er die Frage nahe, unter welchen Bedingungen das Informationsziel erreicht werden kann. Unsere Fallstudie erlaubt in dieser Hinsicht schwerlich abgesicherte Aussagen, jedoch einige Spekulationen, die sich als instruktiv erweisen könnten. In Bayern scheinen die begrenzten Ressourcen der Kampagnenakteure, die Zurückhaltung der Parteien im allgemeinen und die Kampagnenabstinenz der CSU im besonderen, die Konkurrenz medial attraktiver Geschehnisse sowie - zum Teil - die Kompliziertheit des Sachverhalts eine weitere Verbreitung politischer Kenntnisse verhindert zu haben. Die damit verbundenen Ansatzpunkte für Reformvorschläge sind unterschiedlich erfolgversprechend. Das gesellschaftliche Geschehen läßt sich kaum mit Blick auf einen bevorstehenden Volksentscheid gezielt steuern, wie auch politische Parteien schwerlich zu klaren Positionen oder aufwendigen Kampagnen gezwungen werden können. Die Kompliziertheit des Sachverhalts bietet ebenso kaum Ansatzpunkte, es sei denn, man wollte „zu“ komplizierte Gegenstände von direktdemokratischen Verfahren ausschließen und würde klären, wie über den Schwierigkeitsgrad von Regelungsmaterien ent- 
schieden werden sollte. Auch will es wohlüberlegt sein, ob, wie und welche gesellschaftliche Gruppen mit größeren Ressourcen für direktdemokratische Kampagnen ausgestattet werden sollen. Allerdings erscheint es durchaus denkbar, daß Massenmedien intensiver über einen bevorstehenden Volksentscheid berichten und informieren - und womöglich staatliche Stellen relevante Informationen frühzeitig und prominent verbreiten. Über solche Fragen intensiver nachzudenken erscheint geboten, je häufiger direktdemokratische Verfahren zum Einsatz kommen - zumal politische Kenntnisse oder ein Mangel daran durchaus den Ausschlag in die eine oder die andere Richtung geben können.

\section{Anhang: Operationalisierungen und Frageformulierungen}

\section{Abstimmungskampfkontakte}

„Haben Sie in der vergangenen Woche Werbung für oder gegen den Gesetzentwurf „Für echten Nichtraucherschutz!“ in der Presse, im Fernsehen oder im Radio gesehen oder gehört?“ Weiterhin wurde gefragt, ob man mit jemandem gesprochen hat, der für oder gegen den Gesetzentwurf geworben hat, in der letzten Zeit Informationsmaterial wie z.B. Flugblätter, Handzettel, Broschüren oder Postwurfsendungen gelesen, E-Mails oder SMS erhalten, Plakate gesehen oder Internetseiten gelesen hat, auf denen für oder gegen den Gesetzentwurf geworben wurde (Antwortvorgabe „Ja“ [1], „Nein“ [0]).

Interesse am Abstimmungskampf

„Und wie stark interessieren Sie sich speziell für den gerade laufenden Abstimmungskampf zum Volksentscheid über den Nichtraucherschutz in Bayern?“ (fünfstufige Antwortvorgabe von ,überhaupt nicht“ [0] bis ,sehr stark“ [1]).

Politisches Wissen

Tag des Volksentscheids: „Können Sie mir sagen, an welchem Tag der Volksentscheid stattfinden wird?“ 1: 4. Juli; 0: andere Antworten; „,weiß nicht“. 
Die Befragten wurden gebeten, anzugeben, ob die folgenden Aussagen, zum Gesetzentwurf ihrer Meinung nach zutreffen oder nicht zutreffen. „Der Gesetzentwurf verbietet Rauchen in Bierzelten.“ (+), „Bei mehrmaliger Übertretung des Rauchverbots sieht der Gesetzentwurf eine Haftstrafe von bis zu 12 Monaten vor.“ (-), „Nach dem Gesetzentwurf können in Krankenhäusern Raucherräume eingerichtet werden.“ $(+)$, „Wenn in einer Gaststätte geraucht wird, kann nach dem Gesetzentwurf der Raucher bestraft werden, nicht aber der Betreiber der Gaststätte.“ (-), „Wenn jemand verhindern möchte, dass der Gesetzentwurf in Kraft tritt, dann sollte er dagegen stimmen oder nicht an der Abstimmung teilnehmen.“(-).

\section{Akteursbezogenes Wissen:}

Die Befragten wurden gebeten, anzugeben, ob bestimmte Parteien, Vereinigungen und Personen den Gesetzentwurf „Für echten Nichtraucherschutz!“ befürworten, ablehnen oder keine eindeutige Haltung dazu haben. Abgefragt wurde u.a. die Haltung von CSU, SPD, FDP, Grünen, Freie Wählern, ÖDP, Sebastian Frankenberger und des Aktionsbündnisses für Freiheit und Toleranz (AFT).

Die Antworten auf die Wissensfragen wurden entweder als richtig (1) oder falsch (0) kodiert. Für die Regressionsanalyse wurde ein Index aus den Antworten zu CSU, SPD, FDP, Grünen, Freien Wählern und ÖDP gebildet.

Einstellung zum Nichtraucherschutz

Der Index ist der Mittelwert aus folgenden Aussagen (fünfstufige Antwortvorgabe von ,stimme überhaupt nicht zu“ [0] bis ,stimme voll und ganz zu“ [1]).

- „Wie der Nichtraucherschutz jetzt geregelt ist, ist es genau richtig.“ (umgepolt)

- „Das Problem des Nichtraucherschutzes lässt sich durch Raucherräume lösen." (umgepolt)

- In Speiselokalen sollte das Rauchen verboten werden.“

- „Rauchen sollte überhaupt verboten werden.“

- „In Festzelten sollte man rauchen dürfen.“ (umgepolt)

- „Ein totales Rauchverbot in Gaststätten gefährdet die Existenz vieler Gaststätten." (umgepolt)

- „In Kneipen sollte Rauchen erlaubt sein.“ (umgepolt) 
Der Index ist der Mittelwert aus folgenden Aussagen (fünfstufige Antwortvorgabe von „stimme überhaupt nicht zu“ [0] bis „stimme voll und ganz zu“ [1]).

- „Es ist die Pflicht des Staates, Menschen vor gesundheitlichen Risiken zu schützen."

- „Um die Sicherheit der Bürger zu garantieren, sollte der Staat Freiheitsrechte des Einzelnen einschränken.“

- „Der Staat sollte sich nicht in Entscheidungen des Einzelnen darüber einmischen, wie er sein Leben führt.“ (umgepolt)

\section{Raucherstatus}

„Rauchen Sie, wenn auch nur gelegentlich?“ (Antwortvorgabe „Ja“ [1], „Nein“ [0]).

\section{Gaststättenbesucher}

„Nun noch zu ein paar alltäglichen Gewohnheiten: An wie vielen Tagen pro Woche besuchen Sie im Durchschnitt Gaststätten, Restaurants, Cafés oder Kneipen?" Befragte, die mindesten einen Tag in der Woche ausgehen, erhielten den Wert 1 , alle anderen den Wert 0 .

\section{Parteibindung: CSU/FDP}

In Deutschland neigen viele Leute längere Zeit einer bestimmten politischen Partei zu, obwohl sie auch ab und zu eine andere Partei wählen. Wie ist das bei Ihnen: Neigen Sie - ganz allgemein gesprochen - einer bestimmten Partei zu? Und wenn ja, welcher? Befragte, die angaben, sich mit CSU oder FDP zu identifizieren, erhielten den Wert 1 , alle anderen den Wert 0.

\section{Elitensignal}

Zunächst wurde ermittelt, mit welcher Partei sich ein Befragter identifiziert. Anschließend wurde eine Variable konstruiert, die angibt, welchen Standpunkt diese Partei zum Gesetzentwurf „Für echten Nichtraucherschutz!“ aus Sicht der Befragten einnimmt. 
Abstimmungspräferenzen

„Beim Volksentscheid am 4. Juli können Sie für oder gegen den Gesetzentwurf „Für echten Nichtraucherschutz!“ stimmen. Wie werden Sie stimmen: für oder gegen den Gesetzentwurf?“ (Antwortvorgaben „Für den Gesetzentwurf“ [1], „Gegen den Gesetzentwurf“ [0]). 